\title{
Assessing Achievement Motive of American and Israeli Managers: Design and Application of a Three-Facet Measure
}

\author{
Dov Elizur
}

Bar-llan University and The Israel Institute of Applied Social Research

A questionnaire to assess the presence of achievement motive in various populations was developed and its structure analyzed. A facet definition of achievement motive was suggested which provided guidelines for the creation of items and the formulation of hypotheses. The Achievement Motive Questionnaire was administered to 132 U.S. and 114 Israeli middle managers from various public and private organizations. The results support the main hypotheses. An empirical double-ordered conceptual system was obtained which reflects the two facets characteristic to this study: (1) kind of confrontation (being confronted or confronting an answer with a challenge) and (2) time perspective (before, during, or after) relative to task performance. The behavior modalities facet was found to order the conceptual space from instrumental to affective and cognitive modality. The systematic construction of the questionnaire based on the facet definition of achievement motive made it possible to distinguish differences in achievement tendencies between the U.S. and the Israeli samples.

Several years ago, a research project on achievement motive of managers was undertaken with the objective of studying differential levels in this motive in various cultures and subcultures. A tool was needed for assessing the presence of this motive that would be equally applicable to populations in different countries, economic sectors, and other subpopulations.

APPLIED PSYCHOLOGICAL MEASUREMENT Vol. 3. No. 2 Spring 1979 pp. 201-212

() Copyright 1978 West Publishing Co.
The most commonly used techniques for assessing the strength of achievement motive are the Thematic Apperception Test (TAT: McClelland, Atkinson, Clark, \& Lowell, 1953), the French Test of Insight (FTI: French, 1958), and the Iowa Picture Interpretation Test (IPIT: Johnston, 1957). Subscales of inventories with the label "achievement" are also frequently used, such as the Edwards Personal Preference Schedule (EPPS: Edwards, 1954), the Personality Research Form (PRF: Jackson, 1967), and the California Psychological Inventory (CPI: Gough, 1953).

The questionnaire assessment procedures seemed more appropriate for the purpose of this study. In searching the literature, it was found that several imaginative efforts have been made in the direction of constructing a questionnaire for assessing achievement (Hermans, 1979; Mehrabian, 1968). Yet in each of the above attempts, the sample of items was more or less ad hoc. No general guidelines for the inclusion or exclusion of observational items were provided. A more systematic criterion for defining items that pertain to achievement motive was necessary.

The issue of item sampling was particularly important because cross-cultural comparisons were of interest. Thus, the selection of items that were both general and culture-free was an important step in the project. 
One way of tackling this problem is to design separate questionnaires for subpopulations, as done by Mehrabian (1968) for males and females. The disadvantages of this approach are that it requires constructing many questionnaires for each of the populations. It is difficult to compare the scores from different populations, and it is difficult to construct such a questionnaire for an investigator who is not a member of the specific culture.

Another way of tackling this problem is to devise items that are general enough to be culturefree. Such general items, being of a more abstract nature, may be less easily comprehensible to the averăge individual. However, with more educated subjects, this limitation may be disregarded. The latter approach seemed preferable and was therefore applied in this study.

There is a growing interest in developing new and more reliable techniques for the assessment of achievement motive. As Atkinson pointed out, "time is ripe for a renewed attack on the problem of developing better techniques for assessing differences in the strength of achievement motive"' (Atkinson \& Raynor, 1974, p. 16).

An attempt to systematically design a questionnaire for assessing the presence of achievement motive was made in this study. The definition of achievement motive was considered to be of importance; as Weinstein (1969) pointed out, "successful measurement of the need for achievement demands a critical logical analysis of what is meant by the label 'achievement'." The formal approach of facet design (Elizur, 1970; Elizur \& Guttman, 1976; Elizur \& Shye, 1976a; Guttman, 1959; Levy \& Guttman, 1975) was used to define the concept of achievement and thus to facilitate the systematic design of the questionnaire and the formulation of hypotheses about the relations between the definitional framework and the structure of the empirical observations.

\section{Defining Achievement Motive}

A mapping sentence definition of the universe of achievement motive has been developed within the framework of this project and described in detail in Elizur and Shye (1976b) and Shye (1978). The conceptualizations found in the literature (Atkinson \& Feather, 1966; Heckhausen, 1967; Hermans, 1970; Jackson, Ahmed, \& Heapy, 1976; McClelland, 1961) helped to define the content of achievement motive.

The definition of achievement motive hinges on the following three domain facets:

\section{Facet A-Behavior Modality}

Achievement motive is specifically related to the instrumental aspect of behavior. To make surre, the action-relatedness of the items "performance of tasks and assignments" was emphasized in the definition and in each of the questions.

Items in previous questionnaires were found to be related sometimes to the feelings of the respondent-affective attitudes; other questions ask for opinions-cognitive attitudes; and certain others refer to actual behavior-instrumental attitudes. The three behavior modalities comprise a specific conceptual facet in common with various other studies in the behavioral sciences (Elizur, 1970; Elizur \& Guttman, 1976).

\section{Facet B-Type of Confrontation}

Some aspects of achievement motive emphasize the readiness of the individual to confront himself/herself with a challenge and to cope with it, such as coping with difficult versus easy tasks, accepting personal versus shared responsibility, and coping with uncertainty of outcomes.

In contrast to these aspects of achievement, which are monolithic in nature, one finds items that emphasize the readiness of the respondent to consider different aspects of the situation and to match answers to challenges: calculating risks, solving problems, and fulfilling needs.

\section{Facet C-Time Perspective}

An additional classification of achievement items may be made according to the time per- 
spective relative to task performance. Certain aspects of performance are relevant mainly before the task is performed, such as uncertainty and calculating risks. During task performance, the focus of efforts is on coping with difficulty and solving problems. Responsibility and satisfying the need to succeed are basically related to the after-performance stage.

\section{Definition}

Achievement motive may be defined formally in a mapping sentence based on the three facets as presented in Figure 1.

Using the mapping sentence, the universe of achievement motive can be divided into subuniverses created by selecting one element of each facet. Each selection ("structuple") represents a content area which is a subuniverse of the conceptual space of achievement motive according to this definition.

Considering the facets specific to this investigation, Facets $B$ and $C$, the content of their combined elements may be observed by items concerned with the following aspects of achievement:

$b_{1} c_{1}$ to confront oneself with a challenge before task performance-to face uncertainty.

$b_{1} c_{2}$ to confront oneself with a challenge during task performance-to face difficulty.

$b_{1} c_{3}$ to confront oneself with a challenge after task performance-to face personal responsibility.

\section{Figure 1}

Mapping Sentence for the Definition of Achievement Motive

The extent that respondent $(x)$ behaves

A. Behavior Modality

a: . cognitively (prefers)

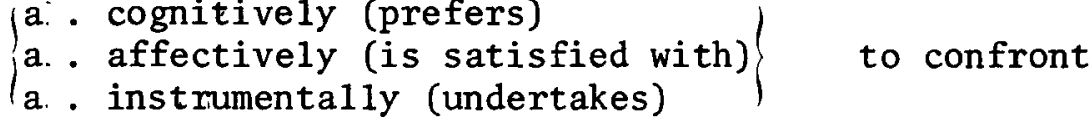

B. Kind of

confrontation

$\left\{\begin{array}{ll}b \cdot \text { himself } \\ \mathrm{b} . & \text { an answer }\end{array}\right\}$

with a challenge associated with the stage

C. Time

Perspective

$\left\{\begin{array}{l}c . \text { before } \\ c . \text { during } \\ \text { c. after }\end{array}\right\}$

task

performance

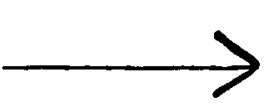

in the sense of the element of Facet A. 
$b_{2} c_{1}$ to confront an answer with a challenge related to the stage before task performance-to take a calculated risk by matching appropriate risk level with an expected gain.

$b_{2} c_{2}$ to confront an answer with a challenge related to the stage during task performance-to match solutions with problems.

$b_{2} c_{3}$ to confront an answer with a challenge related to the stage after task performance-to match an activity to satisfy one's need to succeed.

A geometric portrayal of the definitional framework of achievement motive according to the three facets, as well as the order relations among the elements of Facets $A$ and $C$, is presented in Figure 2.

\section{Objectives and Hypotheses}

The main objective of this study was to examine the structure of achievement motive and to test its stability by comparing two samples from different countries-the U.S. and Israel. In this fashion it was hoped that the proposed lawful relationships would be ascertained and those structural aspects would be isolated that showed the best prospects for being stable across cultures.

The hypotheses tested were (1) the structure of the empirical data would reflect the subdivision of the facets into elements as specified by the mapping sentence and (2) the order of elements within Facet A would correspond to that specified by the mapping sentence; that is, the behavior modalities would be ordered from instrumental to affective and cognitive. In Facet

Figure 2

The Facet Structure of Achievement Motive

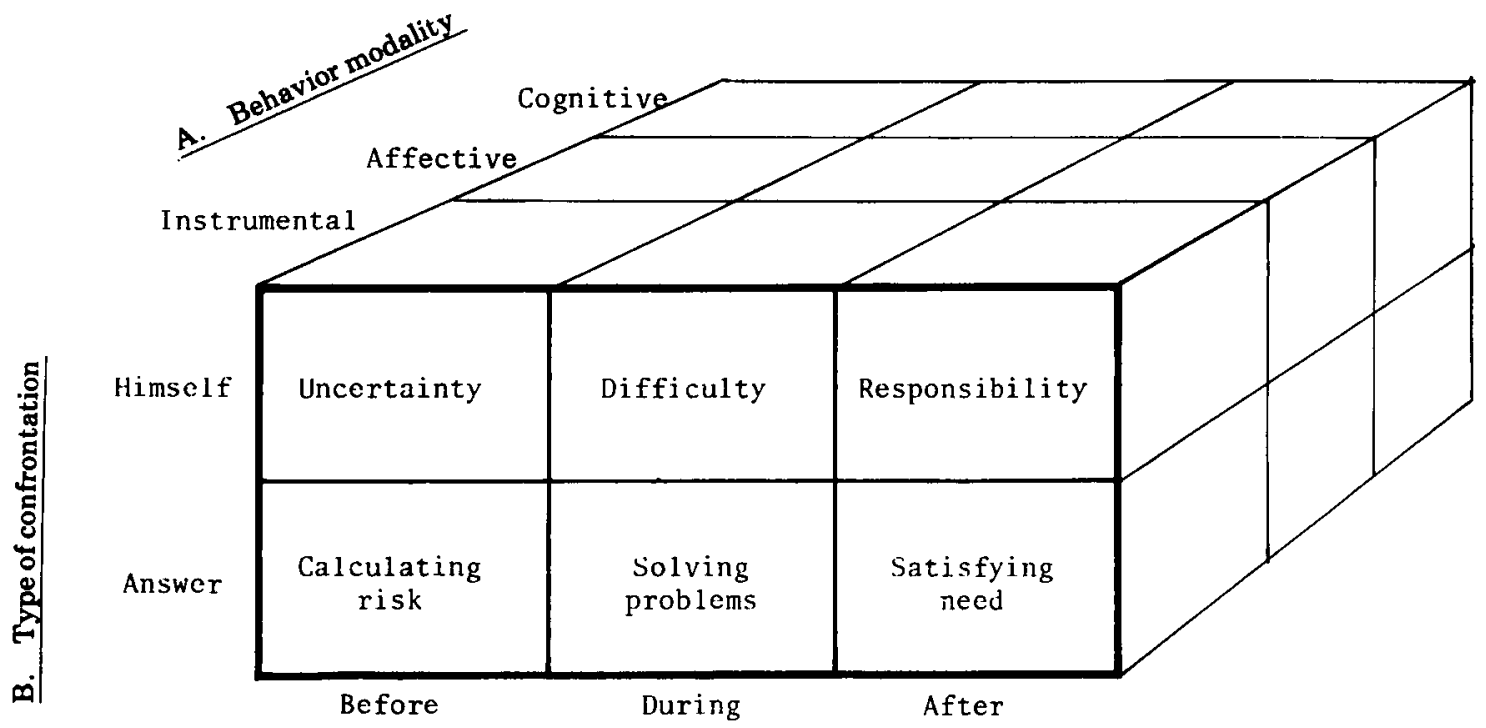

C. Time perspetive relative to task performane 
$\mathrm{C}$, the order would be determined by the time sequence-before, during, and after performance. (Facet $B$ is a dichotomy with no implied order.)

\section{Method}

\section{Subjects}

Data were collected from two samples of middle managers -132 from the United States and 114 from Israel. Each sample represented a relatively diverse group of managers in terms of organizational variables (size, type of organization, department or function) and in terms of personal background (e.g., age, education). The questionnaires were administered to participants in courses or to individuals in the organizations. The samples consisted of managers in both private and public organizations. The U.S. sample, for instance, drew from organizations such as American Pipe Products, National Can Corporation, Pan American World Airways, Staufer Chemicals, Standard Oil, Safeway, Zellerbach Paper Corporation, and the Federal Government General Services Administration. The majority of both samples were male, $77 \%$ in the U.S. sample and $82 \%$ in the Israeli sample. The most frequent age group was 40 to 49 in the U.S. sample and 31 to 39 in the Israeli sample ( $33 \%$ in each).

The two population samples were selected to see whether differential levels of the achievement motive could be distinguished. In the U.S. the private sector is more dominant and affects the public sector; in Israel, on the contrary, the public sector is more dominant and affects the private sector. The private sector is conceived as typically providing more opportunities for entrepreneurial behavior. This research was concerned with exploring whether and in what ways the structure of achievement motive would be different for the two populations under investigation.

\section{The Achievement Motive Questionnaire}

A structured questionnaire for assessing the presence of achievement motive, the Achieve- ment Motive Questionnaire (AMQ), was designed based on the definition of the achievement motive.

The following aspects of achievement motive were distinguished with the aid of Facets B and C:

\begin{tabular}{|c|c|}
\hline $\begin{array}{l}\text { uncertainty } \\
\text { difficulty } \\
\text { responsibility }\end{array}$ & $\begin{array}{l}\left(c_{1}\right) \\
\left(c_{2}\right) \\
\left(c_{3}\right)\end{array}$ \\
\hline $\begin{array}{l}\text { calculating risk } \\
\text { solving problems } \\
\text { satisfying need }\end{array}$ & $\begin{array}{l}\left(c_{1}\right) \\
\left(c_{2}\right) \\
\left(c_{3}\right)\end{array}$ \\
\hline
\end{tabular}

Questions were presented regarding each of the achievement aspects on each of the three behavior modalities (Facet $\mathrm{A}$ ):

Cognitive: preference, e.g., "Do you generally prefer difficult or easy tasks?" (five ranks, from "prefer difficult tasks much more than easy ones" to "prefer easy tasks much more than difficult ones")

Affective: satisfaction, e.g., "Do you generally feel more satisfied when you are personally responsible for a certain task or when you share responsibility with others?" (five ranks, from "I generally feel satisfied with personal responsibility much more than with shared responsibility" to "I generally feel satisfied with shared responsibility much more than with personal responsibility.")

Instrumental: performance, e.g., "Do you usually undertake to perform tasks that require taking calculated risk or tasks whose accomplishment is assured beforehand?" (five ranks, from "I usually undertake to perform tasks requiring calculated risk much more than tasks whose accomplishment is assured" to "I usually undertake to perform tasks whose accomplishment is assured much more than tasks requiring calculated risk.")

\section{Smallest Space Analysis}

Smallest space analysis was used to analyze the relations between the variables and to test 
the structural hypotheses. Smallest space analysis (SSA) is a technique for structural analysis of similarity data (Guttman, 1968; Elizur, 1970; Elizur \& Guttman, 1976). It provides a metric representation of nonmetric information based on the relative distances within a set of points. Each variable is represented by a point in a Euclidean space of one or more dimensions. The points are plotted in the space of smallest pos- sible dimensionality which preserves the rank order of the relations.

The distances among the points are inversely related to the observed relationships among the variables as defined by the correlation coefficients. When the correlation between two variables is high, the distance between them should be relatively small; conversely, when the correlation between two variables is low, the distance

Table 1

Key to Variables in Figures 2-4

\begin{tabular}{|c|c|c|}
\hline $\begin{array}{l}\text { No. of } \\
\text { Variable }\end{array}$ & Content & $\begin{array}{l}\text { The Meaning of the } \\
\text { Positive Direction }\end{array}$ \\
\hline
\end{tabular}

$\left.\begin{array}{rl}1 & \text { Preference of } \\ 7 & \text { Satisfied with } \\ 13 & \text { Undertaking }\end{array}\right\}$

2

8

14

3

9

15

4

\section{5 \\ 11 \\ 17}

$\begin{aligned} 6 & \text { Preference of } \\ 12 & \text { Satisfied with } \\ 18 & \text { Undertaking }\end{aligned}$

tasks involving uncertainty rather than certainty of outcomes

difficult rather than easy tasks

tasks with personal rather than shared responsibility

tasks involving calculated risk rather than no risk or excessive risk

tasks requiring problemsolving rather than application of instructions

tasks gratifying need to succeed rather than ensure avoidance of failure 
between their geometric points should be relatively large.

This method has been successfully applied in various studies to test structural hypotheses (e.g., Elizur, 1970; Elizur \& Guttman, 1976; Elizur \& Shye, 1976a; Levy \& Guttman, 1975).

\section{Results}

Consider first the results pertaining to the two facets specific to this study, Facets B and C. The first two-dimensional projection from the threedimensional SSA computer program reproduced in Figure 3 reflects the structure of the two facets. By observing the map which depicts the structure of the items for the U.S. sample, it is found that the hypotheses were basically supported by the empirical data.
Concerning Facet $C$, the map could be clearly partitioned into three contiguous regions corresponding to the three stages of time perspectives pertinent to task performance. The order of the elements was, as hypothesized, according to the time sequence-before, during, and after performance.

Concerning Facet B, the map could also be clearly partitioned into two distinct regions corresponding to the two characteristics of achievement defined by this facet: "confronting self" and "setting an answer in confrontation with a challenge." The structure of Facets B and C in the Israeli data has been found to conform to that anticipated as illustrated in Figure 2 for these facets. However, in the U.S. sample there was one patent deviation: the items of problem solving were located in the "confronting self" in-

\section{Figure 3}

The Empirical Structure of Achievement Motive.

The First Two dimensional Projection of a Three Dimensional SSA-I.

The U.S. sample, $n=132$; Coefficient of Alienation $=0.12$.

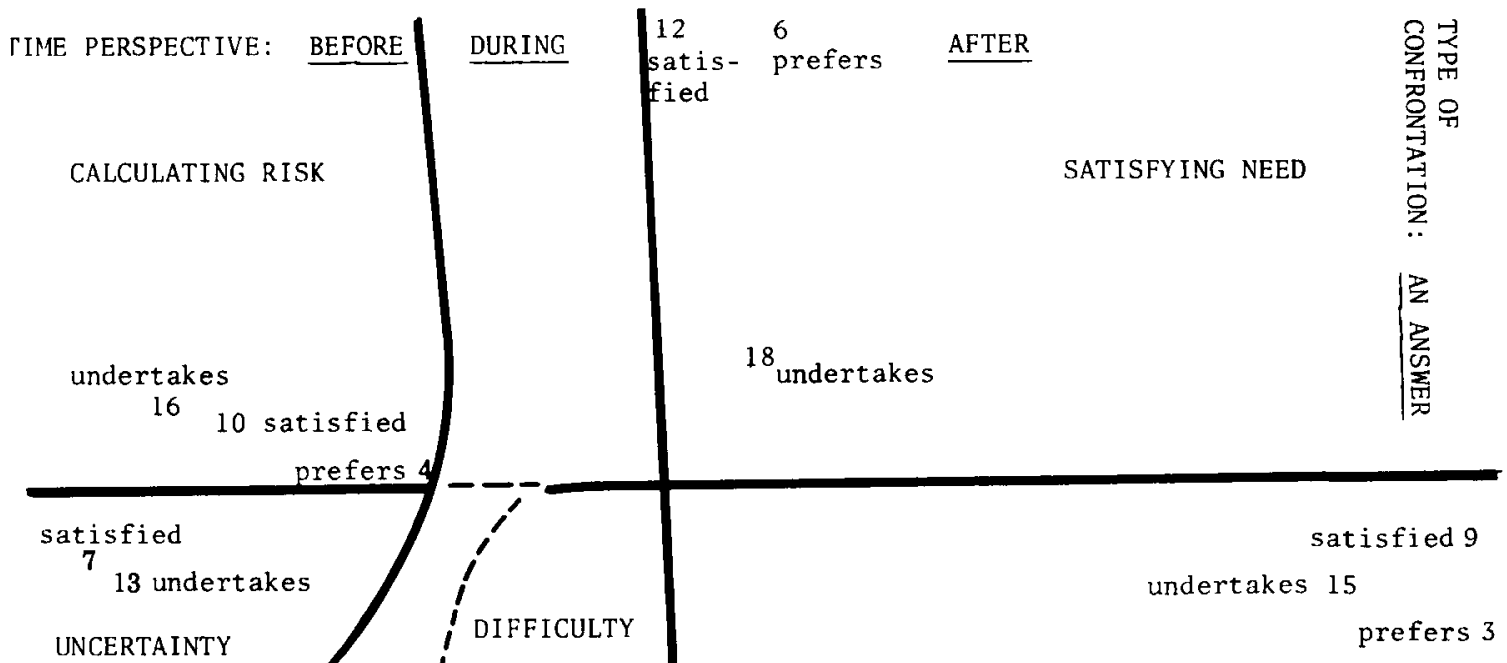

RESPONSIBILITY

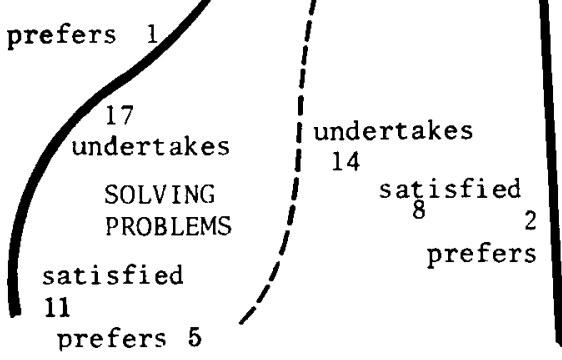


stead of in the "setting an answer in confrontation with a challenge" region of the map, as classified by the mapping sentence and as found in the analysis of the Israeli sample (Elizur \& Shye, 1976b; Shye, 1978). Thus, if the original conceptualization is adhered to, "problem solving" appears to have a more monolithic connotation for the U.S. sample than for the Israeli sample. A possible explanation for this may be that U.S. managers perceive problem solving as a more unitary concept, representing a challenge with which they have to confront themselves, similar to difficulty of task performance. Further research on additional samples may contribute to clarifying this issue.

The difference between the two samples may be interpreted further by the notion that different regions of the map represent different "types of achievement motive." The frequencies of the positive responses to each of the items in the two samples, as presented in Table 2, may be of interest in this context. While only small differences between the two samples could be observed in the after-performance stage of task performance (responsibility and need satisfaction) and moderate differences in the duringtask performance (difficulty and problem solving), remarkably large differences were found in the "before-performance" achievement aspects-uncertainty and calculated risks. These differences may indicate the possibility of distinguishing by this method between different leveis and types of achievement motive among individuals and subpopulations.

No partitioning according to the behavior modality facet was found in the first projection

Table 2

Achievement motive of U.S. and Israeli Middle Managers (percent of the two positive response categories)

\begin{tabular}{|c|c|c|c|c|c|c|c|c|}
\hline \multirow{3}{*}{ Content } & \multirow{3}{*}{ Sample } & \multicolumn{6}{|c|}{ Behavior Modality } & \multirow{3}{*}{$\begin{array}{l}\text { Average } \\
\text { Difference }\end{array}$} \\
\hline & & \multicolumn{2}{|c|}{ Cognitive } & \multicolumn{2}{|c|}{ Affective } & \multicolumn{2}{|c|}{ Instrumental } & \\
\hline & & $\%$ & Diff. & $\%$ & Diff. & $\%$ & Diff. & \\
\hline Uncertainty & $\begin{array}{l}\text { U.S. } \\
\text { Israe1 }\end{array}$ & $\begin{array}{l}50 \\
28\end{array}$ & 22 & $\begin{array}{l}66 \\
37\end{array}$ & 29 & $\begin{array}{l}64 \\
47\end{array}$ & 17 & 23 \\
\hline Difficulty & $\begin{array}{l}\text { U.S. } \\
\text { Israel }\end{array}$ & $\begin{array}{l}82 \\
66\end{array}$ & 16 & $\begin{array}{l}93 \\
81\end{array}$ & 12 & $\begin{array}{l}73 \\
66\end{array}$ & 7 & 12 \\
\hline Responsibility & $\begin{array}{l}\text { U.S. } \\
\text { Israel }\end{array}$ & $\begin{array}{l}85 \\
75\end{array}$ & 10 & $\begin{array}{l}83 \\
84\end{array}$ & -1 & $\begin{array}{l}76 \\
79\end{array}$ & -3 & 2 \\
\hline $\begin{array}{l}\text { Calculating } \\
\text { Risk }\end{array}$ & $\begin{array}{l}\text { U.S. } \\
\text { Israel }\end{array}$ & $\begin{array}{l}76 \\
57\end{array}$ & 19 & $\begin{array}{l}80 \\
59\end{array}$ & 21 & $\begin{array}{l}80 \\
57\end{array}$ & 23 & 21 \\
\hline $\begin{array}{l}\text { Solviag } \\
\text { Problems }\end{array}$ & $\begin{array}{l}\text { U.S. } \\
\text { Israe1 }\end{array}$ & $\begin{array}{l}87 \\
79\end{array}$ & 8 & $\begin{array}{l}89 \\
83\end{array}$ & 6 & $\begin{array}{l}85 \\
80\end{array}$ & 5 & 6.3 \\
\hline $\begin{array}{l}\text { Satisfying } \\
\text { Need }\end{array}$ & $\begin{array}{l}\text { U.S. } \\
\text { Israel }\end{array}$ & $\begin{array}{l}82 \\
75\end{array}$ & 7 & $\begin{array}{l}85 \\
83\end{array}$ & 2 & $\begin{array}{l}78 \\
77\end{array}$ & 1 & 3.3 \\
\hline $\begin{array}{l}\text { Average } \\
\text { Difference }\end{array}$ & & & 13.6 & & 11.5 & & 8 & \\
\hline
\end{tabular}


of the SSA in Figure 3. To find out whether the anticipated order would be found, the other projections of the three-dimensional SSA solution were examined. The third projection of the SSA for the U.S. sample, as shown in Figure 4, clearly reflects the three behavior modalities as defined in the mapping sentence. Encouraged by these results, the third projection of a threedimensional SSA solution of the Israeli sample was examined and an identical structure was found, as can be observed in Figure 5 .

The items located in the central region of the map-the instrumental responses-occupy a relatively smaller space, indicating that they are more strongly related to each other and that the correlations between them are higher. Items dis- persed in the most peripheral region-the cognitive items - on the other side, are more weakly related to each other, thus having lower correlations between them.

It is interesting to note that partition according to the type of confrontation, Facet $B$, is clearly visible in this projection. The order of the structuples, according to Facets B and C, is identical for both samples; however, the order is circular in this projection.

\section{Summary}

In this project, a tool for assessing the presence of achievement motive in various populations was developed and its structure studied.

\section{Figure 4}

The Empirical Structure of Achievement Motive.

The U.S. sample, $n=132$; The Third Two-Dimensional Project of a Three Dimensional SSA-I.

Coefficient of Alienation $=0.12$.

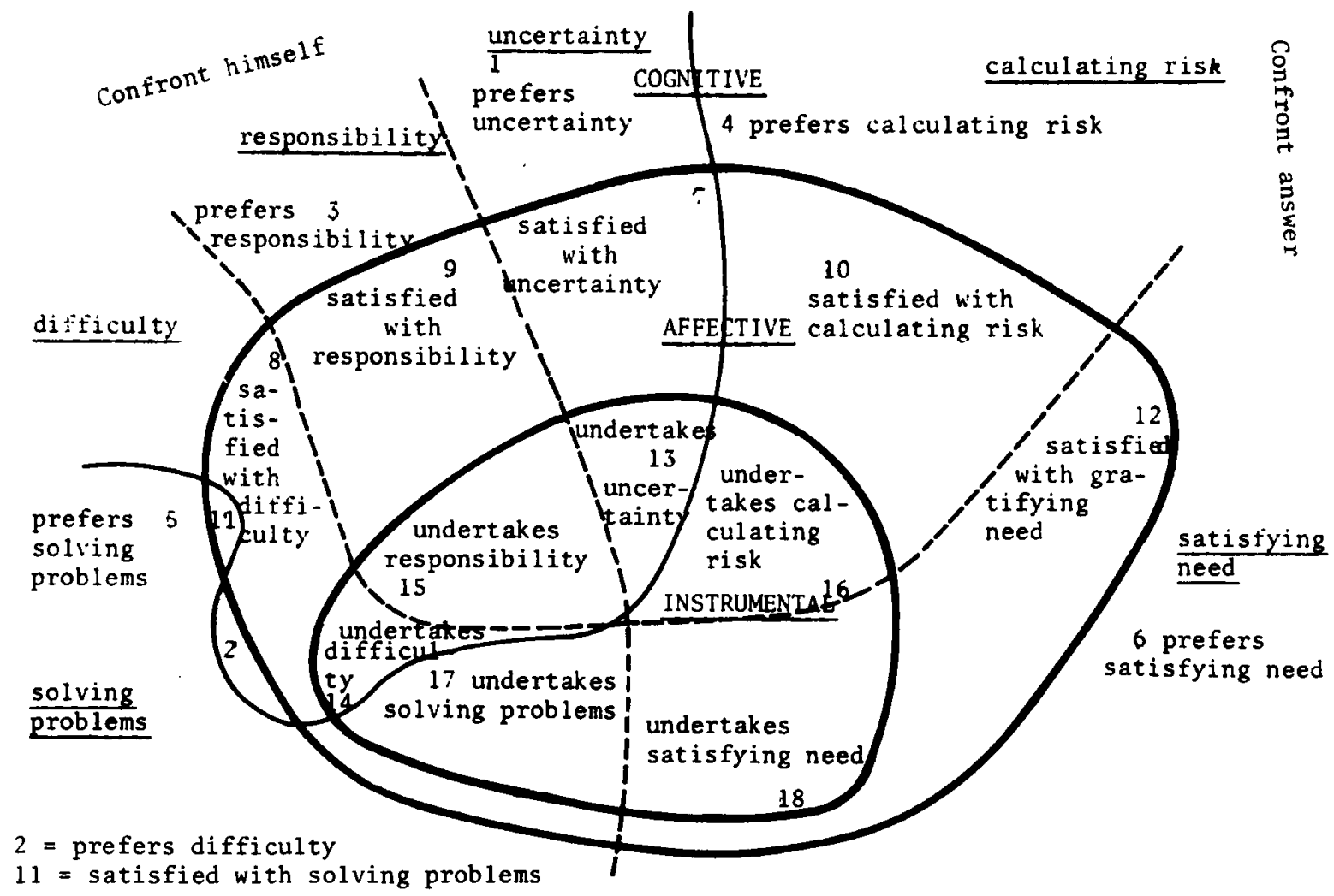


The facet definition of the universe of achievement motive provided guidelines for the creation of items and for the formulation of hypotheses regarding the relations between the definitional framework and the empirical observations.

An empirical double-ordered system, a duplex structure, was obtained in both samples reflecting two of the basic facets of the definition. One facet describes the kind of confrontation "readiness to confront self" or "set an answer in confrontation with a challenge." The other facet describes the time perspective before, during, and after, relative to task performance. The third facet of behavior modalities-cognitive, affective, and instrumental-was found to be re- flected in the third projection of a three-dimensional SSA solution in the predicted order.

Comparison of the two samples revealed generally similar structures except that in the U.S. sample, "problem-solving" items were found to be located in the region of the monolithic items with which the person has to confront himself/herself rather than among the items where the person has to confront an answer with a challenge, suggesting that problem solving may have a more monolithic meaning in English, or at least in the conception of this population sample. The possibility of distinguishing different types of achievement motives was pointed out, supported by the frequency distributions of

\section{Figure 5}

The Empirical Structure of Achievement Motive.

The Third Two Dimensional Projection of a Three Dimensional SSA-I.

The Israeli Sample, $n=114$; Coefficient of Alienation $=0.08$.

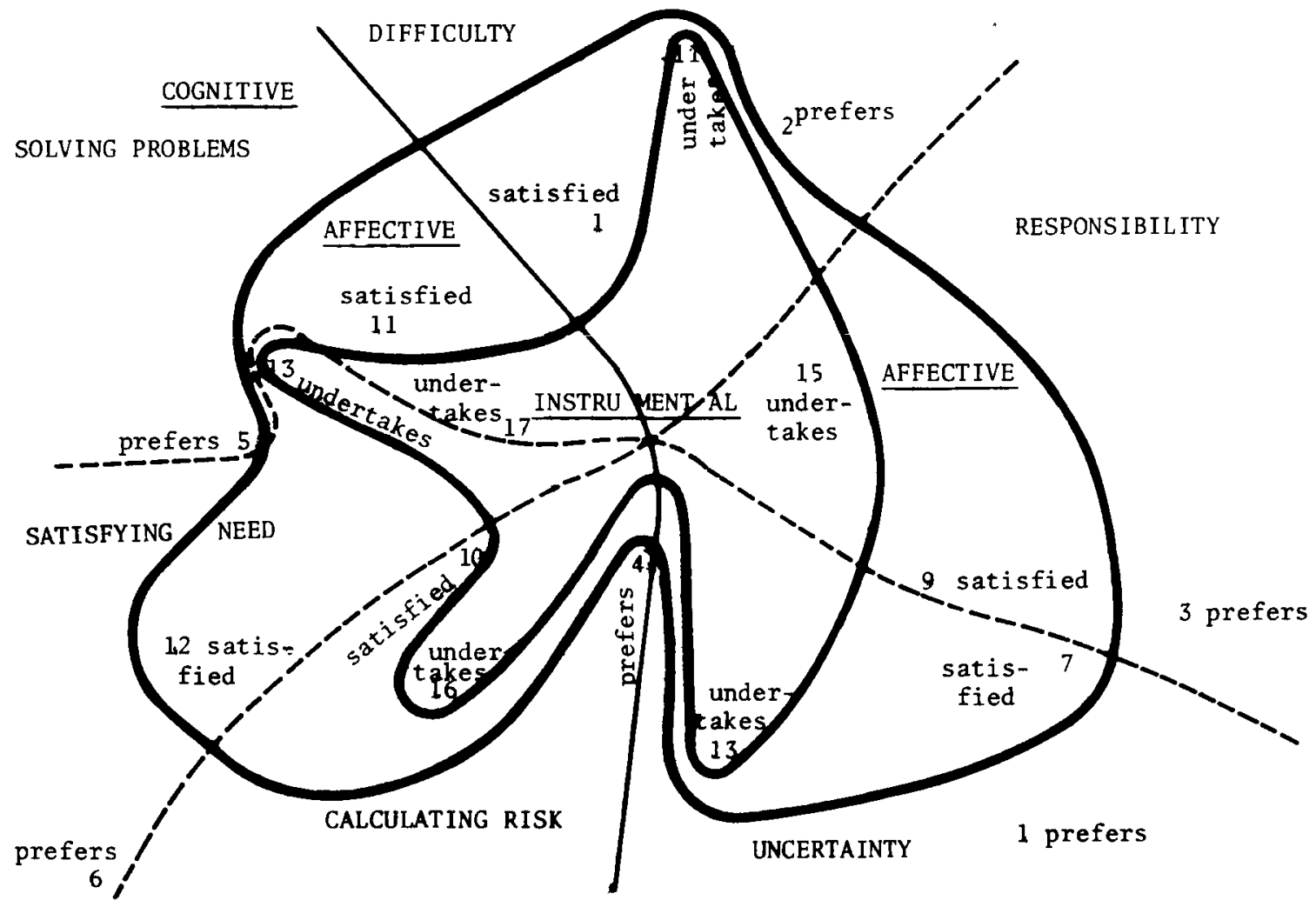


responses in the two samples. The example demonstrates some of the potentialities of the structural design in distinguishing individual and intergroup differences. While the conventional methods of assessing achievement motive usually provide only a single measure, applying facet design provides a set of distinct subuniverses of achievement motive. The Achievement Motive Questionnaire enables the obtaining of observations on each of the subuniverses for individuals or groups investigated. Further research on additional population samples may be instructive in exploring possibilities of distinguishing achievement-motive types and in further elaborating the theoretical and practical utility of the structural definition of achievement motive.

The fact that essentially similar structures were obtained in two independent samples provides substantial support for the suggested definitional framework of achievement motive.

\section{References}

Atkinson, J. W., \& Feather, N. T. A theory of achievement motivation. New York: Wiley, 1966.

Atkinson, J. W., \& Raynor, J. O. Motivation and achievement. Washington, DC: Winston \& Sons, 1974.

Edwards, A. L. Edwards Personal Preference Schedule manual. New York: Psychological Corporation, 1954.

Elizur, D. Adapting to innovation: A facet analysis of the case of the computer. Jerusalem: Jerusalem Academic Press, 1970.

Elizur, D., \& Guttman, L. The structure of attitudes toward work and technological change in an organization. Administrative Science Quarterly. $1976,21,611-622$.

Elizur, D., \& Shye, S. The inclination to re-immigrate: A structural analysis of the case of Israelis residing in France and in the U.S.A. Human Relations, 1976, 29, 1. (a)

Elizur, D., \& Shye, S. [Facet analysis of achievement motive: Defining the concept and analysis of data on Israeli managers.] Jerusalem: Bar-Ilan University and the Israel Institute of Applied Social Research, August 1976. (Hebrew) (b)

French, E. G. Development of a measure of complex motivation. In J. W. Atkinson (Ed.), Motives in fantasy, action, and society. Princeton, NJ: Van Nostrand, 1958.

Gough, H. G. The construction of a personality scale to predict scholastic achievement. Journal of Applied Psychology, 1953, 37, 361-367.

Guttman, L. A structural theory of intergroup beliefs and action. American Sociological Review, 1959, 24, 318-328.

Guttman, L. A general nonmetric technique for finding the smallest coordinate space for a configuration of points. Psychometrika, 1968, 33, 496-506.

Heckhausen, $H$. The anatomy of achievement motivation. New York: Academic Press, 1967.

Hermans, H. J. M. A questionnaire measure of achievement motivation. Journal of Applied Psychology, 1970, 54, 353-363.

Jackson, D. N. Manual for the Personality Research Form. Goshen, NY: Research Psychologists Press, 1967.

Jackson, D. N., Ahmed, S. H., \& Heapy, N. A. Is achievement a unitary construct? Journal of $R^{-}$search in Personality, 1976, 10, 1-21.

Johnston, R. A. A. Methodological analysis of several revised forms of the Iowa Picture Interpretation Test. Journal of Personality, 1957, 25, 283-293.

Levy, S., \& Guttman, L. On the multivariate structure of well-being. Social Indicators Research, 1975, 2, 361-388.

McClelland, D. C. The achieving society. Princeton, NJ: Van Nostrand, 1961.

McClelland, D. C., Atkinson, J. W., Clark, R. A., \& Lowell, E. L. The achievement motive. New York: Appleton-Century-Crofts, 1953.

Mehrabian, A. Male and female scales of the tendency to achieve. Educational and Psychological Measurement, 1968, 28, 493-502.

Shye, S. Achievement motive: A faceted definition and structural analysis. Multivariate Behavioral Research, 1978, 13, 327-346.

Weinstein, M. S. Achievement motivation and risk preference. Journal of Personality and Social Psychology, 1969, 13, 153-172.

\section{Acknowledgments}

This article was written during the author's stay at the Department of Psychology and the Institute of Industrial Relations at the University of California at Berkeley.

The study was supported by the research authorities at Bar-Ilan University and by the Institute of Industrial Relations at the University of California at Berkeley. 
The author is grateful to Samuel Shye for his contribution in the design and analysis of the Israeli part of the project, to Russell Stone for his comments and suggestions, to John Hislop for organizing the data collection in the United States, to Roger Lamm and Howard Rosenberg for administering the questionnaire in their courses, and to Michael Popper for drawing the figures.

\section{Author's Address}

Send requests for reprints or further information to Dov Elizur, Department of Psychology, Bar Ilan University, Ramat-Gan, Israel. 